\title{
Plants used in traditional treatment against anemia in Turkey
}

\author{
Meryem Şeyda ERBAY, Sezin ANIL, Gülay MELİKOĞLU
}

\begin{abstract}
Turkey has many plants used by public for the treatment of various diseases. The usage of these folk medicine which is prepared by these plants has been transferred from generation to generation. Ethnobotanical studies carried out by traditional methods of treatment are recorded and this information is aimed to contribute to the development of the drug. In this study, it
\end{abstract}

has been reached to 77 taxa that is used in traditional treatment against anemia in Turkey and aimed to give information about scientific and local names of these taxa, families, used parts and usage in anemia.

Keywords: Anemia, Medicinal plants, Traditional treatment, Turkey.
Meryem Şeyda ERBAY, Sezin ANIL, Gülay MELIKOĞLU

İstanbul Üniversitesi, Eczacilik Fakültesi, Farmakognozi Anabilim Dalı, 34116, Beyazıt, İstanbul Türkiye

\section{Corresponding author:}

Sezin ANIL

İstanbul Üniversitesi, Eczacıllk Fakültesi, Farmakognozi Anabilim Dal, 34116, Beyaztt, İstanbul Türkiye

E-mail: sezin_kurtoglu@hotmail.com

Phone: 05301749000

Submitted/Gönderilme: 03.03.2016 Accepted/Kabul: 08.04.2016
Revised/Düzeltme: 05.04 .2016

\section{INTRODUCTION}

Anemia is a blood disease which is considered as the reduction of the total number of red blood cells in blood or decrease of their number according to blood volume (hemotocrit) or decrease of their total hemoglobin content (the hemoglobin concentration in the blood ) and can be fairly seen in all countries. It may occur itself or can be the indicative or a symptom of an other underlying disease (1). Anemia may occur because of, chronic blood loss, bone marrow disorders, increased hemolysis, infection, malignancy, endocrine disorders and some other diseases. Many drugs can lead to anemia by causing toxic effect on blood cells, hemoglobin production or erythropoietic organs. Anemia may also occur by the inadequate intake of substances which are necessary for erythropoiesis such as iron, folic acid and vitamin B12 with diet (2).

Anemia, according to the World Health Organization, is defined as being under the hemoglobin concentration of $13 \mathrm{~g} / \mathrm{dL}$ over the age of 15 in men, in women who are not pregnant over the age of 15 and $12 \mathrm{~g} / \mathrm{dL}$, while the pregnancy of $11 \mathrm{~g} / \mathrm{dl}(3)$.

Anemia's common clinical manifestations are weakness, fatigue and paleness. Anemic babies shows themselves to decline irritability, loss of appetite, difficulty swallowing, cry bruising and in pause with development. 
Children with iron deficiency anemia in school performance, especially in fall, understanding and perception difficulties, decreased level of intelligence can be seen. Bone and joint pain, enlarged lymph nodes, liver and spleen size, leukemia and other diseases can be monitored. Palpitations, headache, frequent infections, passing soil to be overcome, plaster, the desire to eat substances such as ice, nail disorders, loss of appetite, loss of taste sensation, pain and hurt the rim of tongue may occur (3).

Anemia prevalence and definition, varies according to demographic and general health status of the region. While discussions on cutoff values used for anemia, under hemoglobin values used in the WHO study was observed in individuals with increased mortality. Many even in apparently healthy individuals in some developing countries are anemic. In countries that are technologically developed, anemia occurs in one in three of the patients admitted to the hospital for any reason. According to the WHO, incidence of anemia in adults is $25 \%$ in Turkey and $14 \%$ in Europe and about 1,022 million adults worldwide are anemic. Yet all over the world in the WHO data covering the years 1993-2005 average of prevalence of anemia were identified as $24.8 \%$, in pregnant women $41 \%, 30 \%$ in non-pregnant women, $12.7 \%$ for males and $23.9 \%$ the elderly. Anemia prevalence in people over age 65 are reported in different studies a wide range varying from $61 \%$ to $3 \%$. These rates varies and show differences according to the characteristics of anemia in the study group (race, age, geography, life and health conditions, etc.) (4).

In anemia treatment, detection of the underlying condition elimination of this condition before the treatment is very important. In anemia due to deficiency of nutritional factors, the defiant iron, folic acid and vitamin B12 can be replaced by oral and injection form.

Anabolic steroids (oxymetholone) can be useful in treatment of hypoplastic and aplastic anemia. Erythropoiesis stimulating agents (eg erythropoietin) can be used in anemia associated with chronic renal failure. Under normal conditions, blood transfusion is not recommended where hemoglobin concentration is not under $7 \mathrm{~g} / \mathrm{dL}$. However, severe anemia can be treated with a transfusion of whole blood (1).

However, because of various side effects seen in synthetic drugs, drug interactions, and higher costs increases the demand for herbal medicines every day. This study reached 77 plant species traditionally used against anemia in Turkey. The information of the plant taxa (scientific and local names, families, used parts and usage in anemia) in this research is given in Table 1. We hope that this study contribute to the development of antianemic drugs.

Table 1. List of the plants used in traditional treatment against anemia in Turkey.

\begin{tabular}{|c|c|c|c|c|c|}
\hline Botanical name & Family & Local name & Plant part used & $\begin{array}{l}\text { Preparation, } \\
\text { administration and } \\
\text { use }\end{array}$ & Reference \\
\hline Achillea millefolium $\mathrm{L}$. & Asteraceae & Civanperçemi & Leaf & Dec., Int. & (5) \\
\hline $\begin{array}{l}\text { Alcea striata (DC.) Alef. } \\
\text { subsp. rufescens (Boiss.) } \\
\text { Cullen }\end{array}$ & Malvaceae & Gülçiçek, Gülhatmi & Flower & $\begin{array}{l}\text { +Milk, } \\
\text { Dec., Int. }\end{array}$ & $(6)$ \\
\hline Arbutus unedo L. & Ericaceae & $\begin{array}{l}\text { Çilek, Dağ çileği, } \\
\text { Sandal }\end{array}$ & Root, Fruit & $\begin{array}{l}\text { Dec. or Inf. Int. } \\
\text { Eaten }\end{array}$ & (7) \\
\hline Artemisia absinthium L. & Asteraceae & $\begin{array}{l}\text { Pelin otu, Acı yavşan, } \\
\text { Acı pelin }\end{array}$ & Aerial part & & (6) \\
\hline A. annua $\mathrm{L}$. & Asteraceae & $\begin{array}{l}\text { Kizılpiren, Püren, } \\
\text { Akpelin }\end{array}$ & Whole plant & & $(8)$ \\
\hline Beta vulgaris L. & Chenopodiaceae & Pancar, Şeker pancarı & Leaf & & (9) \\
\hline $\begin{array}{l}\text { Caltha polypetala Hochst. ex } \\
\text { Laurent }\end{array}$ & Ranunculaceae & $\begin{array}{l}\text { Kral fincanı, Bataklık } \\
\text { nergisi }\end{array}$ & Leaf & $\begin{array}{l}\text { Paultice and tincture, } \\
\text { Int. }\end{array}$ & $(10)$ \\
\hline $\begin{array}{l}\text { Centaurium erythraea Rafn } \\
\text { subsp. erythraea }\end{array}$ & Gentianaceae & $\begin{array}{l}\text { Kirmizikantaron, } \\
\text { Pembekantaron, } \\
\text { Afyonotu }\end{array}$ & Aerial part & & $(11)$ \\
\hline \multirow[t]{2}{*}{ Ceratonia siliqua L. } & Fabaceae & Keçiboynuzu, Harnup & Fruit & Inf., Int. & $(12)$ \\
\hline & & & & Molasses, Int. & $(13,14,15)$ \\
\hline
\end{tabular}




\begin{tabular}{|c|c|c|c|c|c|}
\hline Botanical name & Family & Local name & Plant part used & $\begin{array}{l}\text { Preparation, } \\
\text { administration and } \\
\text { use }\end{array}$ & Reference \\
\hline $\begin{array}{l}\text { Chenopodium album } \mathrm{L} \text {. } \\
\text { subsp. album var. album }\end{array}$ & Chenopodiaceae & Sirken, Aksirken & Leaf, Aerial part & Inf., Int. & (6) \\
\hline Cichorium intybus L. & Asteraceae & Hindiba, Karakavuk & Leaf, Root & Inf., Dec., Int. & $(15)$ \\
\hline Corylus avellana $\mathrm{L}$. & Corylaceae & Fındık, Deli fındık & Seed & Eaten & (9) \\
\hline $\begin{array}{l}\text { C. avellana L. var. pontica (C. } \\
\text { Koch) Winkler }\end{array}$ & Corylaceae & Funduk & Seed & Eaten & $(16)$ \\
\hline $\begin{array}{l}\text { Crataegus azarolus } \\
\text { var. aronia } \mathrm{L} .\end{array}$ & Rosaceae & Alıç & Fruit & Eaten & $(7)$ \\
\hline $\begin{array}{l}\text { Cucurbita moschata } \\
\text { Duchesne cv. }\end{array}$ & Cucurbitaceae & $\begin{array}{l}\text { Bal kabağı, Kaşıkçı } \\
\text { kabağı }\end{array}$ & Seed & +Yoghurt, Fac. & $(17)$ \\
\hline $\begin{array}{l}\text { Dioscorea communis } \\
\text { (L.) Caddickand } \\
\text { Wilkin }\end{array}$ & Dioscoreaceae & $\begin{array}{l}\text { Acı ot, Sarmaşı, } \\
\text { Vicirne, Mıcır, Bıcık } \\
\text { otu, Kapurcuk, } \\
\text { Kediyen }\end{array}$ & $\begin{array}{l}\text { Flowering branch, } \\
\text { Root }\end{array}$ & Raw or Inf., Int. & $(18)$ \\
\hline Diosypros kaki L. & Ebenaceae & Hurma & Fruit & Eaten & (8) \\
\hline $\begin{array}{l}\text { Ecballium elaterium (L.) A. } \\
\text { Rich. }\end{array}$ & Cucurbitaceae & $\begin{array}{l}\text { Eşek hıyarı, Acı kelek, } \\
\text { Delibostan }\end{array}$ & Fruit & Eaten & $(19)$ \\
\hline Ficus carica $\mathrm{L}$. & Moraceae & İncir, Yemiş, Heleş & Fruit & Raw, Int. & $(20)$ \\
\hline Foeniculum vulgare Miller & Apiaceae & Rezene, Arapsaçı & & & $(21)$ \\
\hline Fragaria vesca $\mathrm{L}$. & Rosaceae & Dağ çileği & Fruit & Eaten & $(17)$ \\
\hline $\begin{array}{l}\text { Glycyrrhiza glabra L. var. } \\
\text { glabra }\end{array}$ & Fabaceae & Meyan, Meyan kökü & Root & & (6) \\
\hline Juglans regia L. & Juglandaceae & Ceviz & Leaf, Fruit shell & & $(22)$ \\
\hline Juniperus drupacea Lab. & Cupressaceae & Andız, Andız ağacı & Fruit & Molasses, Int. & (19) \\
\hline $\begin{array}{l}\text { Laurocerasus officinalis } \\
\text { Roemer }\end{array}$ & Rosaceae & Taflan, Karayemiş & Fruit & & $(11)$ \\
\hline $\begin{array}{l}\text { Lavandula stoechas L. subsp. } \\
\text { stoechas }\end{array}$ & Lamiaceae & $\begin{array}{l}\text { Karabaş otu, Karabaş } \\
\text { kekiği, Morbaş }\end{array}$ & Aerial part & & $(14)$ \\
\hline Lepidium sativum L. & Brassicaceae & Tere, Tereotu & Leaf & Dec., Int. & $(23,24)$ \\
\hline L. vesicarium $\mathrm{L}$. & Brassicaceae & Tere otu, Çakçak otu & Leaf & & (6) \\
\hline $\begin{array}{l}\text { Lycopersicon esculentum } \\
\text { Mill. cv }\end{array}$ & Solanaceae & Domates & Fruit & Raw or cooked, Int. & $(17)$ \\
\hline Malus domestica Borkh. & Rosaceae & Elma & Fruit & Molasses, Int. & $(17)$ \\
\hline Malva nicaeensis All. & Malvaceae & Ebegümeci & Leaf & & $(25)$ \\
\hline \multirow[t]{2}{*}{ Melilotus officinalis (L.) All. } & Fabaceae & $\begin{array}{l}\text { Yonca, Sarı yonca, } \\
\text { Kokulu yonca }\end{array}$ & Young leaf & Raw, Int. & (6) \\
\hline & & & Leaf & Inf., Int. & $(26)$ \\
\hline $\begin{array}{l}\text { Melissa officinalis L. subsp. } \\
\text { officinalis }\end{array}$ & Lamiaceae & $\begin{array}{l}\text { Melisa, Oğul otu, } \\
\text { Limon otu }\end{array}$ & $\begin{array}{l}\text { Flowering and } \\
\text { leafy branch }\end{array}$ & & $(27)$ \\
\hline Morus alba L. & Moraceae & Ak dut, Beyaz dut & Fruit & Molasses, Int. & $(25)$ \\
\hline M. nigra L. & Moraceae & Kara dut, Dut & Fruit & $\begin{array}{l}\text { Molasses, jam, syrup } \\
\text { and juice, Int. } \\
\text { Raw, Int. }\end{array}$ & $\begin{array}{l}(8,11,17,25, \\
28,29) \\
(17,24)\end{array}$ \\
\hline Nigella arvensis L. & Ranunculaceae & Çörek otu & Seed & +Honey, Int. & $(30)$ \\
\hline
\end{tabular}




\begin{tabular}{|c|c|c|c|c|c|}
\hline Botanical name & Family & Local name & Plant part used & $\begin{array}{l}\text { Preparation, } \\
\text { administration and } \\
\text { use }\end{array}$ & Reference \\
\hline $\begin{array}{l}\text { Opuntia ficus-indica (L.) } \\
\text { Miller }\end{array}$ & Cactaceae & $\begin{array}{l}\text { Tin sabır, Kaynana } \\
\text { dili, Frenk inciri }\end{array}$ & Fruit & Raw, Int. & $(31)$ \\
\hline Paliurus spina-christi Miller & Rhamnaceaea & $\begin{array}{l}\text { Çaltı, Çakır dikeni, } \\
\text { Karaçalı }\end{array}$ & Seed & Dec., Int. & $(32)$ \\
\hline Papaver rhoeas L. & Papaveraceae & Gelincik & Flower & $\begin{array}{l}\text { Syrup, Int. } \\
\text { Cooked with leaf of } \\
\text { Spinacia oleraceae and } \\
\text { Malva nicaeensis, Int. }\end{array}$ & $\begin{array}{l}(8,33) \\
(8)\end{array}$ \\
\hline Petroselinum crispum Mill. & Apiaceae & Maydanoz & Leaf & Dec., Int. & $(30,34)$ \\
\hline $\begin{array}{l}\text { Phyllitis scolopendrium (L.) } \\
\text { Newn. }\end{array}$ & Aspleniaceae & Geyikdili eğreltisi & Aerial part & Dec., Int. & $(35)$ \\
\hline Polygonum arenastrum Bar. & Polygonaceae & $\begin{array}{l}\text { Madımak, } \\
\text { Kurtpençesi }\end{array}$ & Aerial part & & $(8,23)$ \\
\hline P. aviculare L. & Polygonaceae & $\begin{array}{l}\text { Madımak, Kuşdili, At } \\
\text { mercimeği }\end{array}$ & Aerial part & Inf., Int. & $(6,26)$ \\
\hline P. cognatum Meissn. & Polygonaceae & $\begin{array}{l}\text { Madımak, Solucan } \\
\text { otu }\end{array}$ & Aerial part & Inf., Int. & $(6,26)$ \\
\hline Portulaca oleraceae L. & Portulacaceae & Semizotu & $\begin{array}{l}\text { Aerial part } \\
\text { Fresh Fruit }\end{array}$ & $\begin{array}{l}\text { Dec., Int. } \\
\text { Juice, Int. }\end{array}$ & $\begin{array}{l}(8,10) \\
(36)\end{array}$ \\
\hline $\begin{array}{l}\text { Prunus kurdica Fenzl ex } \\
\text { Fritsch }\end{array}$ & Rosaceae & $\begin{array}{l}\text { Lektır, Momıx, } \\
\text { Mamix }\end{array}$ & Fruit & $\begin{array}{l}\text { Inf., Int. } \\
\text { Eaten }\end{array}$ & (37) \\
\hline $\begin{array}{l}\text { P. spinosa L. subsp. dasyhylla } \\
\text { (Schur) Domin }\end{array}$ & Rosaceae & $\begin{array}{l}\text { Yabani erik, } \\
\text { Çakaleriği, Güvem }\end{array}$ & Fruit & Juice, Int. & $(11)$ \\
\hline Punica granatum L. & Punicaceae & Nar & Fruit & Juice, Int. & $(17,38)$ \\
\hline $\begin{array}{l}\text { Pyrus elaeagnifolia } \\
\text { Pall. }\end{array}$ & Rosaceae & $\begin{array}{l}\text { Yaban armudu, } \\
\text { Yabani armut, Ahlat }\end{array}$ & Fruit & Eaten & $(7)$ \\
\hline $\begin{array}{l}\text { P. elaeagnifolia } \\
\text { subsp. kotschyana } \\
\text { (Boiss. exDecne.) } \\
\text { Browicz }\end{array}$ & Rosaceae & $\begin{array}{l}\text { Yaban armudu, } \\
\text { Yabani armut, Ahlat }\end{array}$ & Fruit & Eaten & (7) \\
\hline Rosa canina L. & Rosaceae & Kuşburnu & Fruit & $\begin{array}{l}\text { Dec., Int. } \\
\text { Inf., Int. }\end{array}$ & $\begin{array}{l}(39,40) \\
(36,37)\end{array}$ \\
\hline & & & Folwer and fruit & Inf., Int. & $(41)$ \\
\hline Rosmarinus officinalis $\mathrm{L}$. & Lamiaceae & Biberiye, Kuşdili & Aerial part & & (14) \\
\hline Rubus canescens DC. & Rosaceae & Böğürtlen & $\begin{array}{l}\text { Fruit } \\
\text { Root and fruit } \\
\text { Young leaf and } \\
\text { flower }\end{array}$ & $\begin{array}{l}\text { Raw, Int. } \\
\text { Dec., Int. }\end{array}$ & $\begin{array}{l}(8) \\
(28)\end{array}$ \\
\hline $\begin{array}{l}\text { R. canescens DC. var. } \\
\text { canescens }\end{array}$ & Rosaceae & $\begin{array}{l}\text { Böğürtlen, Karamuk, } \\
\text { Orman üzümü }\end{array}$ & Fruit & Raw, Int. & $(23,33)$ \\
\hline $\begin{array}{l}\text { R. canescens DC. var. } \\
\text { glabratus (Godron) Davis \& } \\
\text { Meikle }\end{array}$ & Rosaceae & $\begin{array}{l}\text { Böğürtlen, Karantı, } \\
\text { Kapine }\end{array}$ & Fruit & Jam, Int. & $(27)$ \\
\hline R. discolor Weihe et Nees & Rosaceae & Karantı, Karamuk & Fruit & Eaten & $(33)$ \\
\hline R. sanctus Schreber & Rosaceaea & $\begin{array}{l}\text { Böğürtlen, Diken } \\
\text { çileği }\end{array}$ & Fruit & $\begin{array}{l}\text { Eaten, } \\
\text { Inf., Int. }\end{array}$ & $(43)$ \\
\hline
\end{tabular}




\begin{tabular}{|c|c|c|c|c|c|}
\hline Botanical name & Family & Local name & Plant part used & $\begin{array}{l}\text { Preparation, } \\
\text { administration and } \\
\text { use }\end{array}$ & Reference \\
\hline R. saxatilis L. & Rosaceae & Böğürtlen & Fruit, root & Dec., Int. & $(20)$ \\
\hline Rumex acetosella $\mathrm{L}$. & Polygonaceae & $\begin{array}{l}\text { Kuzukulağı, } \\
\text { Ekşikulak, } \\
\text { Kemekulağı }\end{array}$ & Aerial part & Fac., Int. & $(6,29)$ \\
\hline R. conglomeratus Muray & Polygonaceae & Labada, Kuzukulağı & Leaf & & $(8)$ \\
\hline R. crispus L. & Polygonaceae & İlebada, Eşek ilibadası & Leaf & & $(30)$ \\
\hline Solanum melongena L. & Solanaceae & Patlican & Fruit & Eaten & $(44)$ \\
\hline Spinacia oleracea L. & Amaranthaceae & Ispanak & Leaf & Raw or cooked, Int. & $(17)$ \\
\hline $\begin{array}{l}\text { Tamus communis L. subsp. } \\
\text { communis }\end{array}$ & Dioscoreaceae & $\begin{array}{l}\text { Acı ot, Sarmaşık, } \\
\text { Kapırcık }\end{array}$ & Leaf & Eaten & $(17)$ \\
\hline $\begin{array}{l}\text { Teucrium chamaedrys L. } \\
\text { subsp. chamaedrys }\end{array}$ & Lamiaceae & $\begin{array}{l}\text { Mahmude otu, Yavşan } \\
\text { otu, Mayasil otu }\end{array}$ & $\begin{array}{l}\text { Flowering and } \\
\text { leafy branch } \\
\text { Whole plant }\end{array}$ & Inf., Int. & (8) \\
\hline & & & & Dec., Int. & $(28)$ \\
\hline $\begin{array}{l}\text { T. chamaedrys L. subsp. } \\
\text { lydium } \mathrm{O} \text {. Schwarz }\end{array}$ & Lamiaceae & $\begin{array}{l}\text { Bodur otu, } \\
\text { Bodurmahmut } \\
\text { otu, Dalak otu, } \\
\text { Kisamahmutotu }\end{array}$ & Aerial part & Inf., Int. & $(33,45)$ \\
\hline T. polium L. & Lamiaceae & $\begin{array}{l}\text { Acı yavşan, Beyaz ot, } \\
\text { Keselmahmut }\end{array}$ & Aerial part & Dec., Int. & (13) \\
\hline Tilia rubra DC. & Tiliaceae & Ihlamur & Flower, leaf & Dec., Int. & (13) \\
\hline Triticum aestivum L. & Poaceae & Buğday & Seed & Fac., Int. & $(46,47,48)$ \\
\hline T. turgidum Steud. cv. & Poaceae & $\begin{array}{l}\text { Buğday, Sarı buğday, } \\
\text { Makarnalık buğday }\end{array}$ & Spica, fruit & Fac., Int. & $(17)$ \\
\hline T. vulgare L. & Poaceae & Buğday & Seed & Fac., Int. & (19) \\
\hline Urtica dioica L. & Urticaceae & Isirgan & Leaf & $\begin{array}{l}\text { Dec., Int. } \\
\text { Cooked, Int. }\end{array}$ & $\begin{array}{l}(25,49) \\
(8,24)\end{array}$ \\
\hline & & & Aerial part & Dec., Inf., Int. & $(40)$ \\
\hline U. pilulifera $\mathrm{L}$. & Urticaceae & Isırgan, Çakırlı ot & $\begin{array}{l}\text { Seed } \\
\text { Leaf and flower }\end{array}$ & $\begin{array}{l}\text { Dried, Int. } \\
\text { Dec., Int. }\end{array}$ & $(50)$ \\
\hline U. urens L. & Urticaceae & $\begin{array}{l}\text { Isırgan, Akıllı 1sırgan, } \\
\text { Küçük ısırgan otu }\end{array}$ & $\begin{array}{l}\text { Aerial part } \\
\text { Fruit }\end{array}$ & $\begin{array}{l}\text { Dec., Int. } \\
\text { Dec., Int. }\end{array}$ & $\begin{array}{l}(45) \\
(24)\end{array}$ \\
\hline Vitis sylvestris $\mathrm{L}$. & Vitaceae & $\begin{array}{l}\text { Deliasma, Yabaniasma } \\
\text { Beyaz üzüm }\end{array}$ & Fruit & $\begin{array}{l}\text { Molasses, Int. } \\
\text { Eaten }\end{array}$ & $\begin{array}{l}(51) \\
(36)\end{array}$ \\
\hline V. vinifera $\mathrm{L}$. & Vitaceae & Üzüm, Asma & Fruit & Eaten & $\begin{array}{l}(13,17,25, \\
36,37,46,48, \\
49,52,53,54, \\
55,56,57) \\
(12,17,36, \\
37) \\
(8,52,53)\end{array}$ \\
\hline Zea mays $\mathrm{L}$. & Poaceae & Misır, Lazut & $\begin{array}{l}\text { Stylus and corn } \\
\text { cob }\end{array}$ & Dec., Int. & $(58)$ \\
\hline
\end{tabular}

Int: Internal, Dec: Decoction, Inf: Infusion, Fac: The fruits are crushed 


\section{MATERIAL AND METHODS}

This study is prepared by searching thesis at the National Higher Education Center and ethnobotanical studies conducted in various parts of the Turkey with selecting regional plants used for anemia.

\section{RESULTS}

Turkey has many plants used by public for the treatment of various diseases. The usage of these folk medicine which is prepared by these plants has been transferred from generation to generation. Ethnobotanical studies carried out by traditional methods of treatment are recorded and this information is aimed to contribute to the development of the drug.

This study reached 77 plant species traditionally used against anemia in Turkey. These plants are mainly from Rosaceae, Polygonaceae, Lamiaceae, Asteraceae, Poaceae, Fabaceae, Moraceae and Urticaceae families (Figure 1).

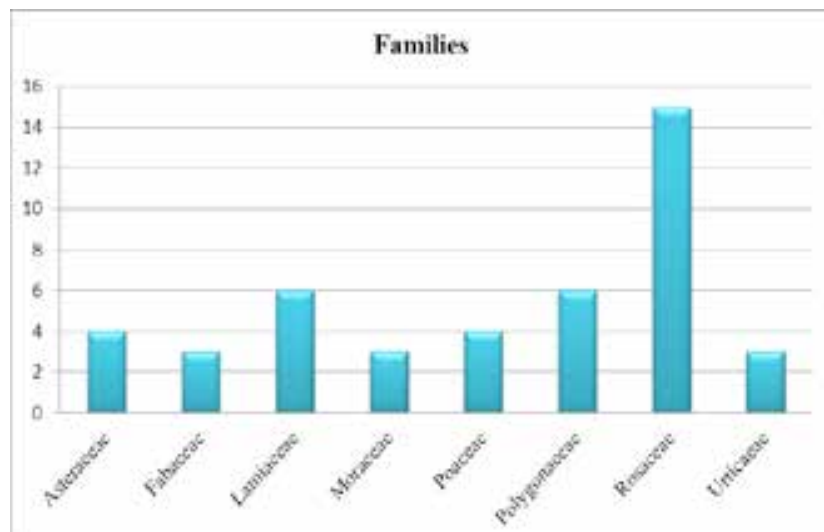

Figure 1. Graph of main families used in traditional treatment against anemia in Turkey.

\section{REFERENCES}

1. Kayaalp SO. Rasyonel Tedavi Yönünden Tibbi Farmakoloji 2. Cilt. 11. Baskı. Hacettepe-Taş Kitapçılık Ltd Şti, Ankara. 2005, pp.1335-1358.

2. Howland RD, Mycek MJ. Lippincott's Illustrated Reviews: Farmakoloji. 3. Baskı. Nobel Tip Kitabevleri, İstanbul. 2009, pp.242.

3. Turkish Society of Hematology. Çocukta Anemi (Kansızlığa) Yaklaşım. Available in: http://www.thd.org.tr/THD Halk/?sayfa=cocuklarda_anemi Accessed: 12.04.2015.

4. Yıldızhan E. Yaşlılarda Anemi Sıklığı ve Özellikleri, Msc Thesis, Düzce: Düzce University, Faculty of Medicine, Deparment of Internal Diseases, 2012.

5. Cakilcioglu U, Turkoglu İ. An Ethnobotanical Survey of
Widely used species against anemia are Vitis vinifera, Urtica dioica, Rubus canescens, Morus nigra, M. alba and Lepidium sativum species. Mainly herbage, leaf and fruit portions of the plants are used in the treatment. The tea form of decoction or infusion from the species which herbage and leaves parts used and the kind of red and purple fruits molasses prepared from ripe fruit are widely used against anemia. Most common substances in these plants chemical compositions are flavonoids, the anthocyanin compounds, tannin, volatile oil, phenolic acids, sugars, minerals (especially iron and phosphorus) and vitamins. In studies about effect of flavonoids on blood components, it was announced that the flavonoids encourage erythropoiesis ( red blood cell formation) (59). On the other side it is though to be that the plants containing iron mineral is useful in iron deficiency anemia. We hope that the information presented in this study can be a source for the development of antianemic drugs.

\section{Türkiye'de Geleneksel Tedavide Kansızlığa Karşı Kullanılan Bitkiler}

\section{ÖZ}

Türkiye'de çeşitli hastalıkların tedavisinde halk tarafından kullanılan birçok bitki bulunmaktadır. Bu bitkiler ile hazırlanan halk ilaçlarının hangi tedavide, nasıl kullanıldığı nesilden nesile aktarılarak günümüze kadar ulaşmıştır. Yapılan etnobotanik araştırmalar ile geleneksel tedavi yöntemleri kayıt altına alınmakta ve bu bilgilerin ilaç geliştirme çalışmalarına katkı sağlaması hedeflenmektedir. $\mathrm{Bu}$ çalışmada Türkiye'de geleneksel tedavide kansızlığa karşı kullanılan 77 taksona ulaşılmış ve bu taksonların bilimsel ve yöresel isimleri, familyaları, kullanılan kısımları ve kansızlıkta kullanılış şekilleri hakkında bilgi verilmesi amaçlanmıştır.

Anahtar kelimeler: Kansızlık, Tibbi bitkiler, Geleneksel tedavi, Türkiye.

Medicinal Plants in Sivrice (Elazı $\breve{g}$ - Turkey). JEthnopharmacol 2010; 132: 165-75.

6. Altundağ E. Iğdır İlinin (Doğu Anadolu Bölgesi) Doğal Bitkilerinin Halk Tarafından Kullanımı. Doktora Tezi, 2009, İstanbul.

7. Sargin SA, Selvi S, Büyükcengiz M. Ethnomedicinal Plants of Aydıncık District of Mersin, Turkey. J Ethnopharmacol 2015; 174: 200-16.

8. Gelse A. Adıyaman ve Çevresinin Etnobotanik Özellikleri. Yüzüncü Yıl Üniversitesi Fen Bilimleri Enstitüsü, Yüksek Lisans Tezi, 2012, Van.

9. Polat R, Cakilcioglu U, Kaltalioğlu K, Ulusan MD, Türkmen Z. An Ethnobotanical Study on Medicinal Plants in Espiye and its Surrounding (Giresun-Turkey). J Ethnopharmacol 2015; 
163: $1-11$

10. Mükemre M. Konalga, Sırmalı, Dokuzdam Köyleri (Çatak/ Van) ve Çevrelerinin Etnobotanik Özellikleri. Yüzüncü Yll Üniversitesi Fen Bilimleri Enstitüsü, Yüksek Lisans Tezi, 2013, Van.

11. Kızılarslan Ç. İzmit Körfezi`nin Güney Kesiminde Etnobotanik Bir Araştırma. İstanbul Üniversitesi Sağlık Bilimleri Enstitüsü, Yüksek Lisans Tezi, 2008, İstanbul.

12. Polat R. Havran ve Burhaniye (Balıkesir) Çevresinde Tarımsal Biyoçeşitlilik ve Etnobotanik Araştırmaları. Balıkesir Üniversitesi Fen Bilimleri Enstitüsü, Doktora Tezi, 2010, Balıkesir.

13. Uysal G. Köyceğiz (Muğla) İlçesinin Etnobotaniği. Muğla Üniversitesi Fen Bilimleri Enstitüsü, Yüksek Lisans Tezi, 2008, Muğla.

14. Gürdal B. Marmaris (Muğla) İlçesinde Etnobotanik Bir Araştırma.İstanbul Üniversitesi Sağlık Bilimleri Enstitüsü, Yüksek Lisans Tezi, 2010, İstanbul.

15. Akgül A. Midyat (Mardin) Civarında Etnobotanik. Ege Üniversitesi Fen Bilimleri Enstitüsü, Yüksek Lisans Tezi, 2008, İzmir.

16. Sağırlığlu M, Arslantürk A, Akdemir Z, Turna M. An Ethnobotanical Survey from Hayrat (Trabzon) and Kalkandere (Rize/Turkey). Int J Biodivers Conser 2012; 5: 31-43.

17. Sargın SA. Alaşehir ve Çevresinde (Manisa) Tarımsal Biyoçeşitlilik ve Etnobotanik Araştırmaları. Balıkesir Üniversitesi Fen Bilimleri Enstitüsü, Doktora Tezi, 2013, İstanbul.

18. Sargin SA, Selvi S, López V. Ethnomedicinal plants of Sarigöl district (Manisa), Turkey. J Ethnopharmacol 2015; 171: 64-84.

19. Güneş S. Karaisalı (Adana) ve Köylerinde Halkın Kullandığı Doğal Bitkilerin Etnobotanik Yönden Araştırılması. Niğde Üniversitesi Fen Bilimleri Enstitüsü, Yüksek Lisans Tezi, 2010, Niğde.

20. Sargin SA. Ethnobotanical Survey of Medicinal Plants in Bozyazı District of Mersin, Turkey. J Ethnopharmacol 2015; 173: 105-26.

21. Özçelik H, Balabanlı C. Burdur İlinin Tibbi ve Aromatik Bitkileri. I. Burdur Sempozyumu, 2005.

22. Güldaş N. Adıyaman İlinde Etnobotanik Değeri Olan Bazı Bitkilerin Kullanım Alanlarının Tespiti. Fırat Üniversitesi Fen Bilimleri Enstitüsü, Yüksek Lisans Tezi, 2009, Elazığ.

23. Gençay A. Cizre (Şırnak)'nin Etnobotanik Özellikleri. Yüzüncü Yll Üniversitesi Fen Bilimleri Enstitüsü, Yüksek Lisans Tezi, 2007, İstanbul.

24. Tuzlacı E, Sadıkoğlu E. Turkish Folk Medicinal Plants, Part VI: Koçarlı (Aydın). İstanbul Ecz Fak Mec 2007; 39: 25-37.

25. Eşen B. Aydınlar Köyü ve Çevresinin (Erdemli/Mersin) Etnobotanik Özellikleri. Selçuk Üniversitesi Fen Bilimleri Enstitüsü, 2008, Konya.

26. Altundağ E, Öztürk M. Ethnomedicinal Studies on The Plant Resources of East Anatolia, Turkey. Procedia Soc Behav Sci 2011;19:756-77.

27. Aktan T. Yenişehir (Bursa) Köylerinin Etnobotanik Özellikleri. Celal Bayar Üniversitesi Fen Bilimleri Enstitüsü, Yüksek Lisans Tezi, 2011, Manisa.

28. Oral DÇ. Konya İlinde Kullanılan Halk İlaçları Üzerinde Etnobotanik Araştırmalar, Gazi Üniversitesi Sağlık Bilimleri
Enstitüsü, Yüksek Lisans Tezi, 2007, Ankara.

29. Yeşilada E, Honda G, Sezik E, Tabata M, Fujita T, Tanaka T, Takeda Y, Takaishi Y. Traditional Medicine in Turkey. V. Folk Medicine in The inner Taurus Mountains. J Ethnopharmacol 1995; 46: 133-52.

30. Deniz L. Uşak University 1 Eylül Kampüsü (Uşak) Florası ve Etnobotanik Açıdan Değerlendirilmesi. Afyon Kocatepe Üniversitesi Fen Bilimleri Enstitüsü, Yüksek Lisans Tezi, 2008, Afyon.

31. Güzel Y, Güzelşemmea M, Miski M. Ethnobotany of medicinal plants used in Antakya: Amulticultural district in Hatay Province of Turkey. J Ethnopharmacol 2015; 174: 118-52.

32. Gürdal B, Kültür Ş. An Ethnobotanical Study of Medicinal Plants in Marmaris (Muğla, Turkey). J Ethnopharmacol 2013; 146: 113-26.

33. Tuzlacı E, Aymaz PE. Turkish Folk Medicinal Plants, Part IV: Gönen (Balıkesir). Fitoterapia 2001; 72: 323-43.

34. Onar S. Bandırma (A1(A), Balıkesir) ve Çevresinin Etnobotaniği. Çanakkale On Sekiz Mart Üniversitesi Fen Bilimleri Enstitüsü, Yüksek Lisans Tezi, 2006, Çanakkale.

35. Saraç DU. Rize İli Etnobotanik Özellikleri. Karadeniz Teknik Üniversitesi Fen Bilimleri Enstitüsü, Yüksek Lisans Tezi, 2013, Trabzon.

36. Sağırlığlu M, Dalgıı̧ S, Toksoy S. Medicinal Plants Used in Dalaman (Muğla), Turkey. J Med Plant Res 2013; 7: 2053-66.

37. Polat R, Cakilcioglu U, Satıl F. Traditional Uses of Medicinal Plants in Solhan (Bingöl-Turkey). J Ethnopharmacol 2013; 148: 951-63.

38. Kayabaşı N. Manyas ve Köylerinde Etnobotanik Bir Çalışma. Balıkesir Üniversitesi Fen Bilimleri Enstitüsü, Yüksek Lisans Tezi, 2011, Balıkesir.

39. Kaval I, Behçet L, Cakilcioglu U. Ethnobotanical Study on Medicinal Plants in Geçitli and its Surrounding (HakkariTurkey). J Ethnopharmacol 2014; 155: 171-84.

40. Mükemre M, Behçet L, Cakilcioglu U. Ethnobotanical Study on Medicinal Plants in Villages of Çatak (Van - Turkey). J Ethnopharmacol 2015; 166: 361-74.

41. Tetik F, Civelek S, Cakilcioglu U. Traditional Uses of Some Medicinal Plants in Malatya (Turkey). J Ethnopharmacol 2013; 146: 331-46.

42. Kural K. Trabzon ve Çevresinde Yayılış Gösteren Faydalı Bitkiler Üzerinde Ekonomik Botanik Yönünden Araştırmalar. İstanbul Üniversitesi Fen Bilimleri Enstitüsü, Yüksek Lisans Tezi, 2012, İstanbul.

43. Hayta S, Polat R, Selvi S. Traditional Uses of Medicinal Plants in Elazı̆ (Turkey). J Ethnopharmacol 2014; 154: 613-23.

44. Güler B, Manav E, Uğurlu E. Medicinal plants used by traditional healers in Bozüyük (Bilecik-Turkey). J Ethnoparmacol 2015; 173: 39-47.

45. Bulut GE. Bayramiç (Çanakkale) Yöresinde Etnobotanik Araştırmalar. Marmara Üniversitesi Sağlık Bilimleri Enstitüsü, Doktora Tezi, 2008, İstanbul.

46. Öztürk M. Nizip Bölgesinin (Aksaray) Florası ve Etnobotanik Özellikleri, Msc Thesis, Konya: Selçuk University, Department of Biology, 2006.

47. Akçiçek E, Vural M. Kumalar Dağı (Afyon) ve Çevresindeki Bazı Bitkilerin Yöresel Adları ve Etnobotanik Özellikleri. Ot Sistematik Botanik Derg 2003; 10: 151-62. 
48. Öztürk M, Dinç M. Nizip (Aksaray) Bölgesinin Etnobotanik Özellikleri. Ot Sistematik Botanik Derg 2005; 12: 93-102.

49. Saday H. Güzeloluk Köyü ve Çevresinin (Erdemli/Mersin) Etnobotanik Özellikleri. Selçuk Üniversitesi Fen Bilimleri Enstitüsü, Yüksek Lisans Tezi, 2009, Konya.

50. Metin A. Mut ve Çevresinde Yetişen Bitkilerin (Mersin) Etnobotanik Özellikleri. Selçuk Üniversitesi Fen Bilimleri Enstitüsü, Yüksek Lisans Tezi, 2009, Konya.

51. Koçyiğit M. Yalova İlinde Etnobotanik Bir Araştırma. İstanbul Üniversitesi Sağlık Bilimleri Enstitüsü, Yüksek Lisans Tezi, 2005, İstanbul.

52. Tekin S. Üzümlü (Erzincan) İlçesinin Etnobotanik Özellikleri. Erzincan Üniversitesi Fen Bilimleri Enstitüsü, Yüksek Lisans Tezi, 2011, Erzincan.

53. Şenkardeş İ. Nevşehir'in Güney İlçelerinde (Acıgöl, Derinkuyu, Gülşehir, Nevşehir-Merkez, Ürgüp) Etnobotanik Araştırmalar. Marmara Üniversitesi Sağlık Bilimleri Enstitüsü, Doktora Tezi, 2014, İstanbul.
54. Ayandın H. Avșar, Şabanözü ve Çile Daği (Polatlı /Ankara) Arasında Kalan Bölgenin Etnobotanik Özellikleri. Selçuk Üniversitesi Fen Bilimleri Enstitüsü, Yüksek Lisans Tezi, 2010, Konya.

55. Han Mİ. Kadışehri (Yozgat) Yöresinin Geleneksel Halk İlacı Olarak Kullanılan Bitkileri. Marmara Üniversitesi Sağlık Bilimleri Enstitüsü, Yüksek Lisans Tezi, 2012, İstanbul.

56. Tuzlacı E, Şenkardeş İ. Turkish Folk Medicinal Plants, X: Ürgüp (Nevşehir). Marmara Pharm J 2011; 15: 58-68.

57. Vural M, Karavelioğulları FA, Polat H. Çilek Dağı (Kırşehir) ve Çevresinin Etnobotanik Özellikleri. Ot Sistematik Botanik Derg 1997; 4: 117-24.

58. Koyuncu O, Yaylacı ÖK, Tokur S. Geyve (Sakarya) ve Çevresinin Etnobotanik Açıdan İncelenmesi. Ot Sistematik Botanik Derg 2009; 16: 123-42.

59. Bilaloğlu GV, Harmandar M. Flavonoidler. Aktif Yayınevi., İstanbul. 2006, pp. 2. 